\title{
Detection of the third class of gamma-ray bursts: magnetar giant flares.
}

\author{
Michela Negro $^{a, b, *}$ and Eric Burns ${ }^{c}$ \\ ${ }^{a}$ NASA Goddard Space Flight Center \\ Greenbelt (MD), USA \\ ${ }^{b}$ University of Maryland Baltimore County, \\ ${ }^{c}$ Louisiana State University \\ E-mail: mnegro1@umbc.edu, ericburns@lsu.edu
}

Around 11.4 million years ago a young, highly magnetized neutron star called magnetar, in the Sculptor galaxy released an enormous amount of energy in the form of a giant flare. On April 15th 2020, some of the emitted photons were detected by a number of gamma-ray telescopes around Earth and Mars. While the analysis of this event, GRB 200415A, was interesting in its own right, it resulted in broader implications for both magnetar and gamma-ray burst (GRB) science. The resulting population study of magnetar giant flares (MGFs), led to the unambiguous identification of a distinct population of 4 local $(<5 \mathrm{Mpc})$ short GRBs. While identified solely based on alignment to nearby star-forming galaxies, their rise time and isotropic energy release are independently inconsistent with the larger short GRB population at $>99.9 \%$ confidence. These properties, the host galaxies, and non-detection in gravitational waves all point to an extragalactic MGF origin. The inferred volumetric rates for events above $4 \times 10^{44} \mathrm{erg}$ of $R=$ $3.8_{-3.1}^{+4.0} \times 10^{5} G p c^{-3} y r^{-1}$ place MGFs as the dominant gamma-ray transient that have been detected from extragalactic sources. As previously suggested, these rates imply that some magnetars produce multiple MGFs, providing a source of repeating GRBs. The rates and host galaxies favor common core-collapse supernova as key progenitors of magnetars.

$37^{\text {th }}$ International Cosmic Ray Conference (ICRC 2021)

July 12 th - 23rd, 2021

Online - Berlin, Germany

\footnotetext{
${ }^{*}$ Presenter
} 


\section{Prologue}

This proceeding is based on a recent paper published in Ap. J. letters (2021) by E. Burns et al. ([1]), to which we refer for a detailed discussion of the results. The above-mentioned paper is a study of the short gamma-ray burst detected population to search for events consistent with a non-cosmological origin (namely neutron star mergers). This study was triggered by the event GRB 200415A, a short gamma-ray burst detected by several instruments on board different satellites (GBM (Fermi), Wind (Konus), BAT (Swift) and Mars-Odyssey (HEND)). The InterPlanetary network (IPN) enabled a precise localization $\left(\sim 20 \mathrm{arcmin}^{2}, 3\right.$ sigma uncertainty) of the event, which aligned with NGC 253, also known as Sculptor galaxy [2]. The significance of the spatial alignment ([1]) and analyses of the time and spectral evolution of this burst ([2], [4]), which differ from typical short gamma-ray bursts, led to the conclusion of it being originated from a magnetar giant flare in the Sculptor Galaxy. Even though not relevant for the work presented here, it is important mention that a high energy gamma-ray emission was also detected by the Fermi-LAT and associated to the same magnetar giant flare ([3]), opening new scenarios for these kind of events.

\section{Introduction}

Magnetars are highly magnetized, young neutron stars that display a wide range of radiative activity, like glitches, bursts, quasi-periodic oscillations, giant flares (see e.g. [5] for a recent review). As of 2004, magnetar giant flares (MGFs) had been observed twice in our Galaxy [6, 7], and once from our largest satellite galaxy, the Large Magellanic Cloud (LMC) [Evans et al. 1980]. MGFs are characterized by a total energy release of over $10^{44} \mathrm{erg} \mathrm{s}^{-1}$ in the X-ray and soft gammaray band. Given this extremely large energy release these events, when extragalactic in origin, they would appear as short GRBs[7] and should be detectable by active gamma-ray burst monitors. Indeed in 2005 and 2007, two extragalactic MGFs were observed (see e.g [8, 9]) and associated to M81 and M83 galaxies. Some previous population studies [10-12], even without identifying additional events of the kind, set lower and upper limits on the fraction of detected short GRBs with MGF origin: this fraction ranges between 1 and $8 \%$.

GRB 200415A added a new member to the detected MGF population, hence now counting 3 Galactic (including the LMC event) and 3 extragalactic events, and triggered our new population study, whose details are described in the next section, while the results are discussed in the last section of this proceeding.

\section{Data samples and analysis}

Short GRB sample. We combined the short GRB sample detected by several GRB monitors (CGRO-BATSE, Konus-WIND, Swift-BAT and Fermi-GBM), additionally exploiting information from the IPN data. Our final GRB selection consisted into 250 short GRB (according to the standard definition of T90<2 s), with measured bolometric $(1 \mathrm{keV}-10 \mathrm{MeV})$ fluences at Earth and a good localization $\left(90 \%\right.$ confidence area $\left.<4.125 \mathrm{deg}^{2}\right)$.

Local Galaxy sample. We used the $\mathrm{z}=0$ Multiwavelength Galaxy Synthesis (z0MGS) Catalog, whic combines GALEX ultraviolet observations and WISE infrared observations. We also 
supplemented the missing information with the the Census of the Local Universe (CLU), which additionally provides the angular size of the galaxies (extended sources are modeled as ellipses). We selected $>100,000$ galaxies between $0.5 \mathrm{Mpc}$ (excluding the Milky Way and its satellite galaxies) and $200 \mathrm{Mpc}$ (way beyond where MGFs can be detected). For each galaxy we made use of their position (RA, DEC and distance), their angular extent and their star formation rate (SFR) as described in the following paragraph.

Likelihood analysis. The goal is to estimate the probability that a given position in the sky (where a short GRB happened) is to produce a MGF with a particular fluence at Earth. We pixeled the sky using HEALPix format sky maps with NSIDE=8192 (pixel width of $\approx 0.5 \mathrm{arcmin}$ ) and the above mentioned probability is defined as

$$
\Omega=4 \pi \sum_{i} \frac{P_{i}^{G R B} P_{i}^{M G F}}{A_{i}}
$$

where the sum runs over the pixels, $A_{i}$ is the pixel area, $P_{i}^{G R B}$ is the GRB probability distribution function at the $i^{t h}$ sky position (given by the localization uncertainty of the GRB). $P_{i}^{M G F}$ is the probability that a given position is to produce a MGF with a particular fluence at Earth, defined as

$$
P_{i}^{M G F}=\operatorname{SFR}_{i} \operatorname{PDF}\left(E_{i s o}\right)
$$

where $\operatorname{PDF}\left(E_{\text {iso }}\right)$ is the probability distribution function of the MGF intrinsic energetic $\left(E_{\text {iso }}\right)$ modeled as a power law whose index we estimated to be $\alpha=1.3 \pm 0.9$ at $90 \%$ confidence iterating over the $E_{\text {iso }}$ values of the previously known MGFs (excluding the first event in the LMG, given the different IPN calibration).

Figure 1 shows the inverse cumulative $\Omega$ function: the blue shaded regions correspond to the null hypothesis (GRB have a non-local origin) and is obtained randomly rotating the galaxy sample in the sky and repeating the $\Omega$ computation; the orange line correspond to the real short GRB sample: there are four events that deviate form the background at more than 5 sigma, unambiguously identifying a new class of GRBs. The light curves and the IPN localizations for the four identified short GRB are shown in Figure 2: one it the recent event GRB 200415A (from the Sculptor galaxy), GRB 051103 and GRB 070201 (associated to M81 and M31 respectively) are two of the previously known extragalactic MGFs; our approach additionally found a new event, GRB 070222, associated to M83, which brings to 7 the population of detected MGFs.

The four events differ from cosmological short GRBs by having significantly shorter rising time and several order of magnitude lower $E_{i s o}$. Figure 3 we show the 4 MGFs (orange) compared to the cosmological short GRB population (blue) a chart $E_{i s o}$ vs rise time. In the same chart we report the same parameters for the galactic MGFs (gray).

Additional analyses We used the entire population (excluding the first 1979 event, because of the different IPN sensitivity), to constrain the intrinsic energetic power law index. To do that we simulated a large number of extragalactic MGFs assigned to a specific host galaxy following a power-law intrinsic energetic distribution with index $\alpha$. The MGFs that could be detected are those whose sampled $E_{i s o}$ and distance produce a flux greater than our detection threshold. We used a Anderson-Darling k-sample test to compare the detected simulated populations to the real one (4 extragalactic MGFs). We scan a wide range of $\alpha$ and find that $\alpha=1.7 \pm 0.4$ is the best range to reproduce our observations. 
We used the intrinsic energetic PDF and the number of detected events to estimate the intrinsic rate of MGFs in the local universe: we find an intrinsic volumetric rate for events above $4 \times 10^{44}$ erg of $R=3.8_{-3.1}^{+4.0} \times 10^{5} G p c^{-3} y r^{-1}$, which places MGFs as the dominant gamma-ray transient that have been detected from extragalactic sources.

We further discuss our results in the next section.

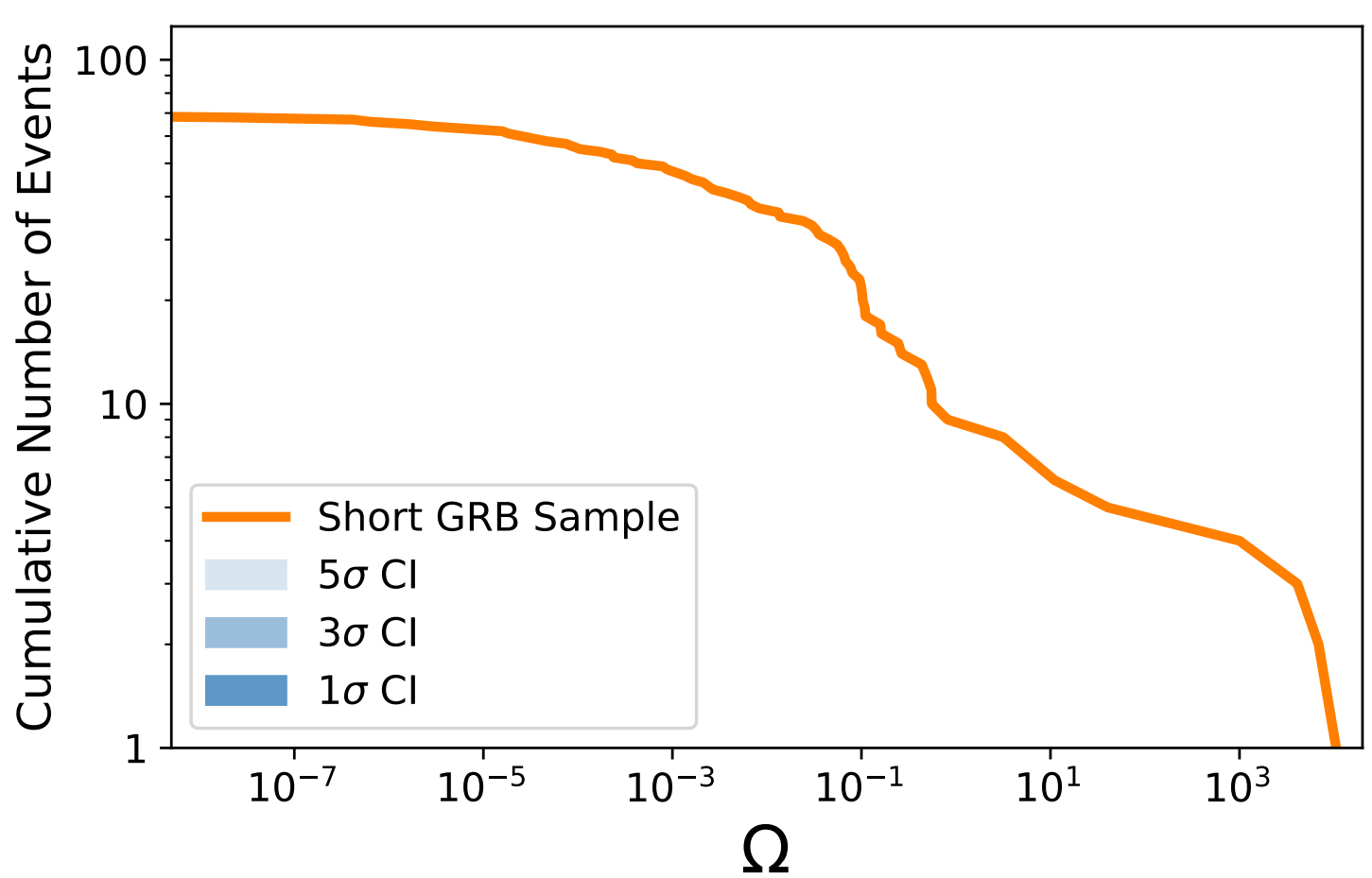

Figure 1: $\Omega$ is a statistic that ranks how believable the event is to be an extragalactic MGF, with values for the true population is shown in orange. The background confidence intervals at 1,3 , and $5 \sigma$ are shown in blue. The four most significant events together surpass $5 \sigma$ discovery significance.

\section{Results and Discussion}

We found a sample of 4 short GRBs that are incompatible with a non-local origin at more than $99.9 \%$ ( $>5$ sigma) confidence. these short GRB are extremely short (a few milli-seconds) and show an intrinsic energetic 5 order of magnitude lower than typical SGRB powered by neutron star mergers. The inferred volumetric rate places MGFs as the dominant gamma-ray transients that can be detected at extragalactic distances. On the other hand we only detected 7 in the past four decades. The explanation to this can be found in the atypical spectrum of these short GRBs: with respect to GRB with cosmological origin, the prompt emission of there events (modelled as a comptonized spectrum) show a higher peak energy (1.5 MeV compared to 0.6 for typical short GRBs) and a greater spectral index ( $\sim 0$ compared to $\sim 0.4$ for typical short GRBs) [4]. This is enough to move 

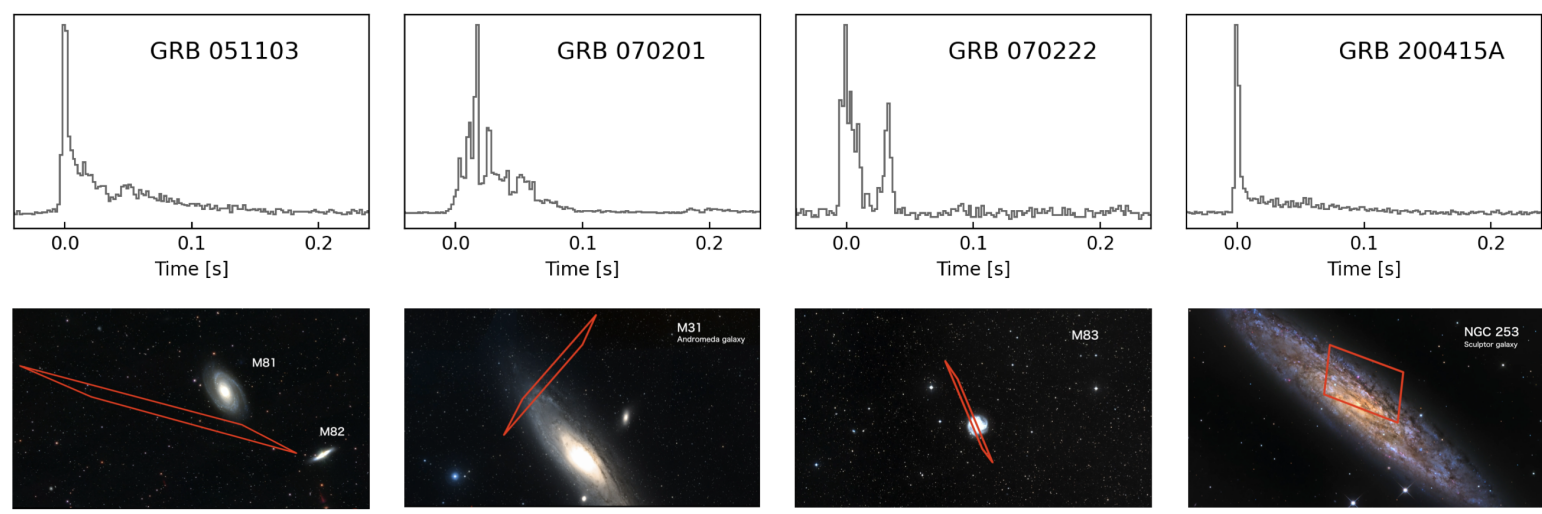

1 in 70,000

1 in 10,000

1 in 130,000

1 in 230,000

Figure 2: Light curves and localizations for the four identified short GRB that show incompatible with a cosmological origin. The numbers below each event is the chance allignment.

the peak of the emission away from the trigger range of current GRB monitors, as shown in Figure 3 (right panel).

The estimated volumetric rate and the type of host galaxies (with high star formation rates), favor core-collapse supernova (CCSN) as the dominant formation channel for magnetars, with at least $0.5 \%$ of CCSN resulting in magnetars. At the same time, the result suggests that some magnetars produce multiple MGFs: this would be the first known source of repeating GRBs.

The newly identified MGF, GRB 070222, shows two temporally and spectrally distinct pulses, which suggest varied behavior of these events and need for further studies and observations.
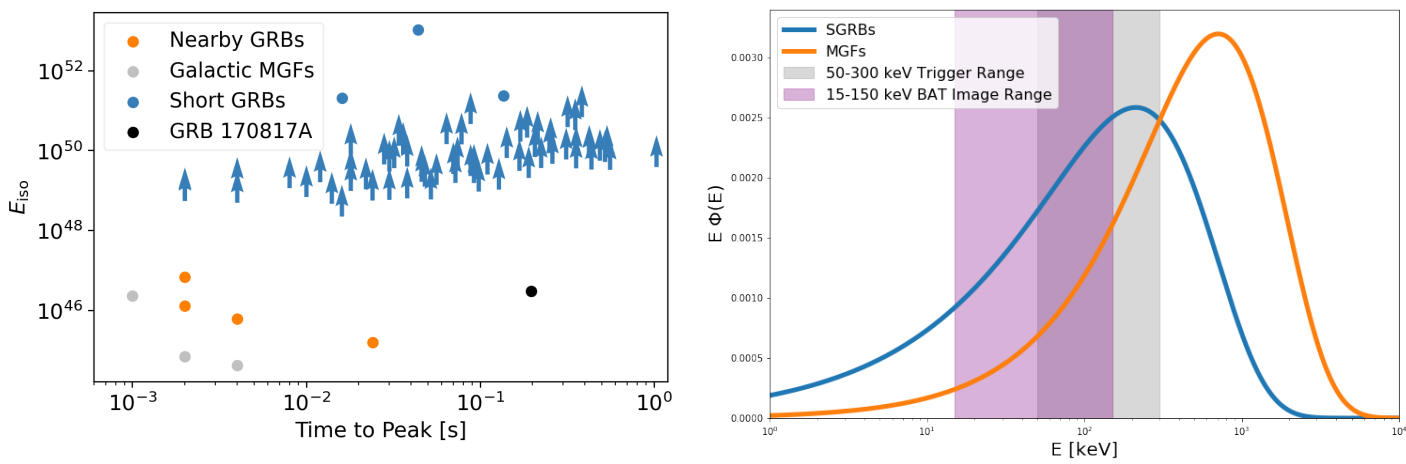

Figure 3: Left: $E_{i s o}$ vs time to peak for the 4 identified extragalactic MGFs (orange), the galactic MGFs (gray) and the cosmological short GRB population (blue). Right: Comptonized spectra for a typical short GRB of cosmological origin (blue) and for GRB 200415A (orange) of MGF origin.

\section{Epilogue}

Despite the fact that the idea of a subsample of short GRB have a MGF origin is not new, our study unambiguously detect a class of short GRB incompatible with a cosmological origin (neutron 
star mergers) at more than 5 sigma, a significance level rarely achieved in astrophysics. The identified class of short GRBs are compatible with the expectation of having MGFs as progenitors.

We would like to acknowledge all the authors of the paper in Ref [1], who contributed to this result. In particular: Dimitry Svinkin, Kavin Hurley, Zorawar Wadiasingh, Georges Younes, Rachel Hamburg, Anna Ridnaia and David Cook.

\section{References}

[1] E. Burns et al. ApJL, 907(2):L28, 2021.

[2] D. Svinkin et al. Nature, 589(7841): 211-213, 2021.

[3] The Fermi-LAT Collaboration. Nature Astronomy, 2021.

[4] O. J. Roberts et al. Nature, 589(7841):207-210, 2021.

[5] V. M. Kaspi et al. ARA\&A, 55:261-301, 2017.

[6] Hurley, K., Cline, T., Mazets, E., et al. 1999a, Nature, 397, 41

[7] Hurley, K., Boggs, S., Smith, D., et al. 2005, Nature, 434, 1098

[8] Ofek, E. O., Kulkarni, S., Nakar, E., et al. 2006, Astrophys J., 652, 507

[9] Mazets, E. P., Aptekar, R. L., Cline, T. L., et al. 2008, Astrophys. J., 680, 545

[10] Popov, S. B., Stern, B. 2006, Mon. Not. R. Astron. Soc., 365, 885

[11] Ofek, E. O. 2007, Astrophys. J., 659, 339

[12] Svinkin, D. S., et al. 2015, Mon. Not. R. Astron. Soc., 447, 1028 